\title{
Visual Experience Is Required for the Development of Eye Movement Maps in the Mouse Superior Colliculus
}

\author{
Lupeng Wang, ${ }^{1,2}$ Mingna Liu, ${ }^{1}$ Mark A. Segraves, ${ }^{1}$ and $\odot$ Jianhua Cang ${ }^{1}$ \\ ${ }^{1}$ Department of Neurobiology and ${ }^{2}$ Interdepartmental Neuroscience Program, Northwestern University, Evanston, Illinois 60208
}

\begin{abstract}
Topographic maps are a fundamental feature of the brain's representations of the sensory environment as well as an efficient way to organize motor control networks. Although great progress has been made in our understanding of sensory map development, very little is known about how topographic representations for motor control develop and interface with sensory maps. Here we map the representation for eye movements in the superior colliculus (SC) in awake mice. As stimulation sites were sampled along the anterior-posterior axis, small amplitude, nasally directed (ipsiversive) saccadic eye movements were evoked by microstimulation in anterior SC, followed by a smooth progression to large, temporally directed (contraversive) movements in posterior SC. This progressive change of movement amplitude and direction is consistent with the global polarity of the retinotopic map in the superficial SC, just as in primates and cats. We then investigated the role of visual experience in the development of eye movement map by studying mice reared in complete darkness. Saccades evoked by SC stimulation as well as spontaneous saccadic eye movements were larger in the dark-reared mice, indicating that visual experience is required to fine-tune the gain of saccades and to establish normal eye movement maps in the SC. Our experiments provide a foundation for future studies to investigate the synaptic organization and developmental mechanisms of sensorimotor transformations in mice.
\end{abstract}

Key words: electrical microstimulation; saccades; superior colliculus; topographic maps

Significance Statement

The superior colliculus (SC) is a midbrain structure important for multisensory integration and sensorimotor transformation. Here we have studied eye movement representations in the SC of mice, a species that has become a popular model in vision research because of available genetic tools. Our studies show mice make saccadic eye movements spontaneously and in response to SC stimulation. The mouse SC contains an eye movement map that has the same global polarity as the overlaying visual map, just like in cats and primates. Furthermore, we show that visual experience is required for establishing the normal eye movement map. Our study provides a necessary basis for future mechanistic studies of how SC motor maps develop and become aligned with sensory maps.

\section{Introduction}

Normal brain functions require a precise patterning of the connections in the nervous system. To direct gaze toward a visual target, for example, the visuomotor system depends upon precise sensorimotor transformations in the midbrain superior colliculus (SC) and then to premotor and motor nuclei controlling eye

Received Jan. 11, 2015; revised June 25, 2015; accepted July 29, 2015.

Author contributions: L.W., M.A.S., and J.C. designed research; L.W. and M.L. performed research; L.W., M.L., M.A.S., and J.C. analyzed data; L.W., M.L., M.A.S., and J.C. wrote the paper.

This work was supported by National Institutes of Health Grant (R21EY023060 to J.C. and M.A.S.). We thank Dr. Tomoya Sakatani and Dr. Dan Dombeck for technical help and members of the Cang lab for insightful discussion. The authors declare no competing financial interests.

Correspondence should be addressed to either Jianhua Cang or Mark A. Segraves, Department of Neurobiology, Northwestern University, Evanston, IL 60208, E-mail: cang@northwestern.edu or m-segraves@northwestern.edu.

L. Wang's present address: Laboratory of Sensorimotor Research, National Eye Institute/National Institutes of Health, Bethesda, MD 20892.

DOI:10.1523/JNEUROSCI.0117-15.2015

Copyright $\odot 2015$ the authors $\quad 0270-6474 / 15 / 3512281-06 \$ 15.00 / 0$ and skeletal muscles (Gandhi and Katnani, 2011). To meet this demand, the retinotopic map in the superficial layers of the primate and cat SC is in topographic register with the motor map in the intermediate and deep layers (referred to collectively here as deep layers), such that deep layer activity directs gaze to spatial locations represented by overlying superficial sites (Schiller and Stryker, 1972). This unique organization makes the mammalian SC an excellent model for studying the interactions between sensory and motor representations during development.

Despite the great progress that has been made in our understanding of sensory map development, including retinotopic representation in the superficial SC (Cang and Feldheim, 2013), how topographic representations for motor control are established is not well understood (Stein, 1984). Furthermore, very little is known about how sensory and motor maps are aligned during development. In the SC, where the topography of sensory and motor representations is closely aligned, visual input may instruct the development of the eye movement map in the deep 

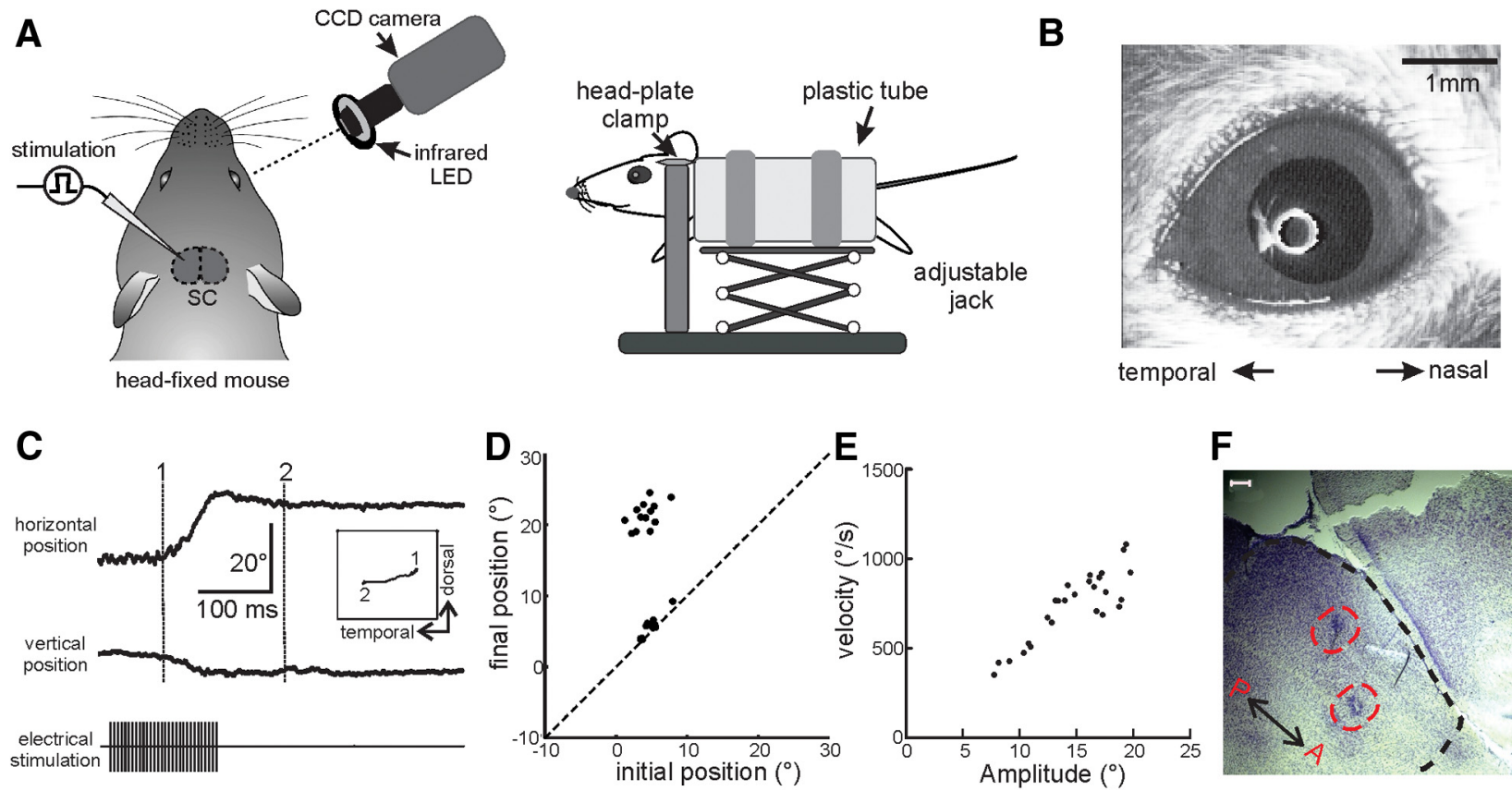

temporal $\leftarrow$

Figure 1. Electrical stimulation in deeper layers of the SC evokes saccadic eye movements in mice. $\boldsymbol{A}$, Schematic diagram of the experimental setup. $\boldsymbol{B}$, An example image of the eye. The white circle in the pupil is the reflection of a circular array of infrared LEDs. C, An example of SC-evoked rapid eye movement in temporal (top trace) and ventral (bottom trace) direction. Inset depicts the eye's trajectory from its initial ("1") to final ("2") position, with the numbers corresponding to the dotted lines in the main plot. $\boldsymbol{D}$, Comparison of initial and final eye positions for a single SC stimulation site. Each point marks horizontal eye position for a single trial. Points near the unity line are trials that failed to evoke any movement. All trials presented here used the same stimulation parameters. $\boldsymbol{E}$, Correlation between evoked eye movement amplitude and peak saccadic velocity. Data points from multiple stimulation sites were included. $\boldsymbol{F}$, Nissl staining of a sagittal section of the SC (outlined by the black dashed line). The red circles mark the electrolytic lesion sites in two different penetrations. Note that lesions do not necessarily mark where the lowest thresholds for evoking saccades were found. The anterior (A)-posterior(P) direction is labeled. Scale bar, $100 \mu \mathrm{m}$.

layers. Alternatively, the eye movement map might develop independently of visual experience and the potential influence of the overlying visual map. Surprisingly, such a fundamental question has not been investigated in mammals, although a single report found that the motor map in the optic tectum of barn owls develops abnormally when visual input is prevented during development (du Lac and Knudsen, 1991).

One reason for this lack of progress is that no motor map has yet been revealed in the SC of the mouse, a species where the repertoire of available genetic tools has been useful in studying visual system development. Recent behavioral and physiological studies have demonstrated that the mouse visual system has better perceptual capacities than previously thought and it possesses most of the functional features seen in higher mammals (Huberman and Niell, 2011). For eye movements, optokinetic movements have been observed in mice and are likely mediated by genetically identified retinal ganglion cells that project to the accessory optic system (Yonehara et al., 2009; Dhande et al., 2013). In contrast, it remains to be determined whether the mouse SC contains a motor map for saccadic eye movements.

In this study, we set out to reveal the organization of the eye movement map in the mouse SC, and to examine the influence of visual experience upon its development. We found that the mouse SC indeed contains a motor map similar to that in higher mammals. In mice with normal visual experience, the direction and amplitude of horizontal eye movements evoked by deep layer stimulation is consistent with the progressive shift of visual receptive fields of superficial layer locations along the anterior-posterior axis. In contrast, mice deprived of visual experience during postnatal development have a degraded motor map and larger spontaneous eye movements, indicating that the formation of a normal SC motor map requires normal visual experience.

\section{Materials and Methods}

Animal preparation. All animals were used in accordance with protocols approved by Northwestern University Institutional Animal Care and Use Committee. Adult wild-type C57BL/6 mice of both genders were housed either under $12 \mathrm{~h}$ light/dark cycle (normal reared, NR) or in constant darkness from birth until the day of recording $\sim 2$ months later (dark reared, DR). Each mouse underwent a survival surgery under isoflurane anesthesia (1-3\% in $\mathrm{O}_{2}$; supplemented with $2.0 \mathrm{mg} / \mathrm{kg}$ dexamethasone). A small metal bar was attached to the skull using MetaBond (Parkell), so that the head could be held still during experiments. In addition, a small craniotomy was made over the cortex above the SC $(0-1.5 \mathrm{~mm}$ lateral from the midline suture and $0-1 \mathrm{~mm}$ anterior from the lambda suture). The craniotomy was then covered by silicone elastomer (Kwik-Sil; WPI) until the time of the experiment, one to a few days later. After surgery, carprofen $(5 \mathrm{mg} / \mathrm{kg})$ was administered to reduce pain and prevent inflammation. The mice were then housed individually under the same light/dark cycle they experienced before the surgical procedure.

Two preparations of awake head-fixed mice were used. In bodyrestrained experiments, the mouse was positioned in a plastic tube (25 $\mathrm{mm}$ diameter) with its head held by miniature clamps via the metal bar. In body-mobile experiments, the mouse was positioned on top of a Styrofoam ball floating on pressured air (5-10 psi) as adapted from Dombeck et al. (2007). The mouse was still head fixed, but could sit, run, and attempt to make left/right heading turns on the ball. Each recording session was completed within $4 \mathrm{~h}$ to prevent fatigue. After the final experiment for each individual, the mouse was killed by an intraperitoneal injection of Euthasol $(150 \mathrm{mg} / \mathrm{kg})$ and perfused with PBS and then $4 \%$ PFA solution.

Monitoring eye position. A high-speed, $400 \mathrm{~Hz}$ CCD camera (UNIQ Vision) was used to monitor eye position in the head-fixed mice (Sakatani and Isa, 2004). We imaged an area of $4 \times 3 \mathrm{~mm}$ with a macro lens (Edmund Optics). An infrared-light-emitting diode (LED) ring (740 $\mathrm{nm}$ ) was used to illuminate the eye. A LabView program (Sakatani and Isa, 2004, 2007) calculated the center and boundary of the pupil from the 

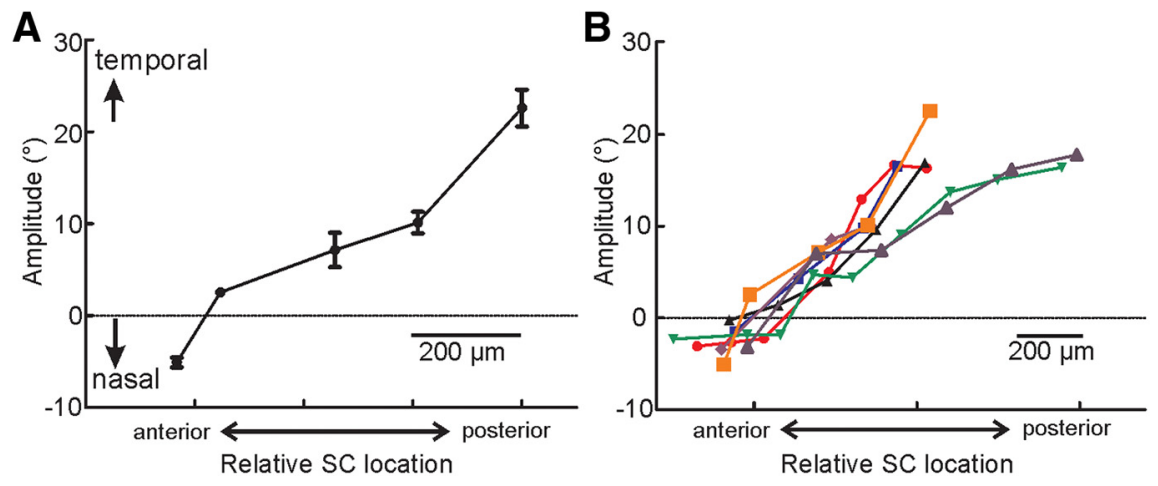

Figure 2. Eye movement maps in the mouse SC. A, Data from an example mouse showing the progressive change of right eye movement amplitude and direction while moving stimulation sites along the anterior-posterior axis of the SC. Data are presented as mean \pm SEM. $\boldsymbol{B}$, Population plot of horizontal eye movement amplitude as a function of the position of stimulation sites. Individual lines of different colors are data from single animals $(n=7)$, aligned to their intercept at zero evoked movement when fitted to a straight line.
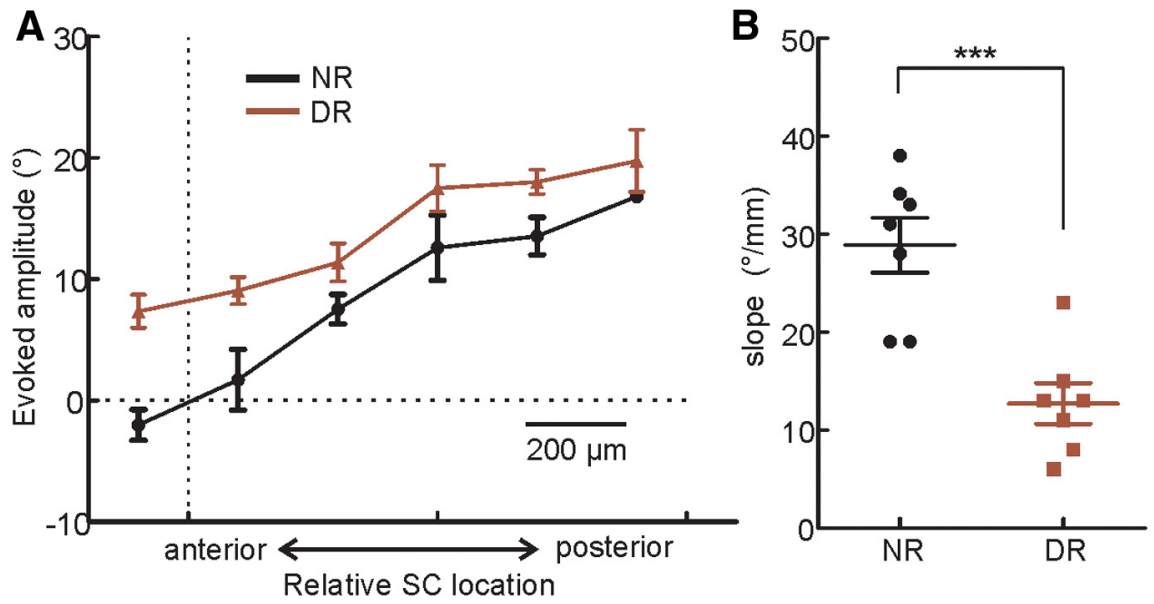

Figure 3. Visual experience is required for the normal development of eye movement maps in the mouse SC. A, Horizontal amplitudes of evoked saccades in DR (brown) and NR (black) mice. The visual receptive fields of the superficial SC were mapped for some penetrations to determine the $\mathrm{SC}$ location that represented the vertical meridian, which was used to align maps from different mice. $\boldsymbol{B}$, Eye movement maps in DR mice had a smaller slope along the anterior-posterior axis than in the NR mice ( $p<$ $0.001, t$ test). Each data point represents the slope from an individual animal. Pooled data were presented as mean \pm SEM.

captured images, and the pupil displacement in the $2 \mathrm{D}$ image was converted to a rotation angle based on the estimated eyeball radius $(1.25$ $\mathrm{mm})$ for adult C57BL/6 mice. The reflection of the LED ring in the eye provided a reference point for the eye position and was set as a $0^{\circ}$ angle during each recording session. Because mice lack a fovea, the $0^{\circ}$ point was estimated by centering the LED ring at the natural resting position of the eye for each mouse, which was kept constant for a given experiment. The raw values of eye position angles were used only in Figure $1 E$, and all analyses were for saccade amplitude, which is the difference between eye positions before and after saccades, independent of how the reference point was set.

Electrical stimulation and extracellular recording. Single tungsten microelectrodes (FHC) were used to record and stimulate in the SC. The electrodes were inserted vertically through the craniotomy and the overlaying cortex. The SC superficial layers $(1-1.5 \mathrm{~mm}$ below the cortical surface) were identified following our published procedure (Wang et al., 2010). The locations of stimulation and recording sites were further confirmed and evaluated by electrolytic lesion and post hoc Nissl staining (Fig. $1 F$ ). Microstimulation was achieved by a Grass S- 88 stimulator and a pair of PSIU-6 stimulus isolation units using biphasic, negative first current pulses, $0.2 \mathrm{~ms}$ duration per pulse, at frequencies of $200 \mathrm{~Hz}$ for $100-200 \mathrm{~ms}$. The amount of stimulus current, usually between 40 and 60 $\mu \mathrm{A}$, was monitored by measuring the voltage drop across a $1 \mathrm{~K} \Omega$ resistor in series with the electrode. All electrical signals were acquired using a
System 3 workstation (Tucker Davis Technologies) at $3 \mathrm{KHz}$ and analyzed off-line.

Data analysis and statistics. We identified stimulation-evoked saccades as eye movements evoked within $200 \mathrm{~ms}$ after the onset of microstimulation and with peak velocities exceeding 100 degrees/s. Similarly, selfgenerated, spontaneous eye movements were considered saccades if their velocity exceeded 100 degrees/s. In all analysis, the eye position traces were downsampled at $200 \mathrm{~Hz}$. The velocity trace was smoothed using a moving $45 \mathrm{~ms}$ median filter and each saccade was inspected manually to remove artifacts, such as blinking. To quantify eye movement amplitude, the initial eye position was determined as the mean of the eye position during a $50 \mathrm{~ms}$ time window before the saccade. The final position was determined as the mean of the eye position during a $50 \mathrm{~ms}$ time window following (and including) the time point when the maximum change in eye position was evoked. The amplitude of eye movement was calculated as the difference between the initial and final eye positions. Nasotemporal and dorsoventral movements were analyzed separately. All eye movement recordings were made using the right eye, and, by convention, temporal/rightward and dorsal/upward movements were set as positive. The eye velocity was calculated for each time point and its maximum value was designated as the peak velocity of the saccade evoked by a given stimulus.

All values are presented as mean \pm SEM. Statistical analyses and graph plotting were done with Prism (GraphPad Software) and MATLAB (The MathWorks).

\section{Results}

Microstimulation in the SC reliably evokes rapid saccadic eye movements in awake mice

We used head-fixed mice to simplify monitoring and analysis of eye movements (Fig. $1 A, B$; see Materials and Methods for details). Electrodes were inserted vertically through the overlaying cortex, and the SC superficial layers were identified by visually evoked responses in local field potentials and multiunit activity (Wang et al., 2010). The locations of stimulation sites were further confirmed and evaluated by electrolytic lesion and post hoc Nissl staining (Fig. $1 F$ ). Brief trains of current $(10-60 \mu \mathrm{A}, 0.2 \mathrm{~ms}$ per pulse, $200 \mathrm{~Hz}$ for 100-200 ms) were delivered at different depths. When the microstimulation was delivered in the deep layers of the SC, rapid saccadelike eye movements were evoked (Fig. 1C). The evoked eye movements often contained both horizontal (nasotemporal) and vertical (dorsoventral) components, although the nasotemporal components were generally of greater amplitude (Fig. $1 C$ ). After each evoked eye movement, the eye usually stayed at its new position for $>1 \mathrm{~s}$ before returning to its original position. The eye movements that were evoked from a single site were of similar amplitude, and displayed in an all or none fashion when stimulation was near threshold (Fig. 1D). Finally, when analyzing eye movements of different amplitudes, we observed an almost linear correlation between movement amplitude and peak velocity (Fig. 1D), producing the typical "main sequence" seen for saccades in humans and monkeys (Bahill et al., 1975; Dias and Segraves, 1999). 
We then systematically changed the sites of stimulation to study whether the mouse SC contains a map of saccade amplitude. We kept the stimulation current near threshold and constant for each animal (usually between 40 and $60 \mu \mathrm{A}$ ). For each penetration, only trials at the first depth where reliable eye movements could be evoked were used for quantification. This was done to reduce the variations caused by the fact that most of the penetrations were not perfectly perpendicular to the SC surface. The means of the movement amplitudes of these trials were calculated for each penetration, and these values were then plotted against the relative SC location for the corresponding penetrations in a given experimental session (Fig. 2A). Because the eye movements were generally very small along the dorsoventral axis in the head-fixed mice, we focused our analysis on the nasotemporal component of the evoked saccades. The nasotemporal axis of the visual space is represented along the anterior-posterior axis in the superficial layers of the mouse SC. We thus moved our stimulation site along this axis. For increasingly more posterior stimulation sites, the evoked movement changed direction from nasal to temporal and increased in amplitude (Fig. $2 ; n=7$ ). This progressive change of movement amplitude and direction is consistent with the global polarity of the retinotopic map in the superficial SC. For example, nasally directed eye movements were evoked when stimulating the anterior pole of the SC, which represents part of ipsilateral visual field in mice (Dräger and Hubel, 1976). In other words, these results demonstrate the existence of a topographic map for saccade amplitude in the deep layers of the mouse SC. This eye movement map has the same global polarity with the overlying retinotopic map, just like in cats and primates.

\section{Visual deprivation degrades the eye movement map}

To determine whether the establishment of the eye movement map in the mouse SC requires visual experience, we reared mice in complete darkness from before eye opening until adulthood (postnatal day $\sim 60$ ), and then studied the organization of SC eye movement maps in these mice. To provide points of reference that would allow us to combine data from different animals, we mapped the visual receptive fields of the superficial SC for some penetrations using multiunit responses to small flashes of light squares, following our published procedure (Wang et al., 2010). Like in the NR mice, the SC of the DR mice included both contralateral and partial ipsilateral visual field representations. We therefore determined the SC location that represented the vertical meridian of the visual field by extrapolation for each mouse, and used it for aligning data from different mice and for comparing maps between the two groups.

As was the case for NR mice, microstimulation of the SC in the DR mice still evoked rapid saccadic eye movements. However,
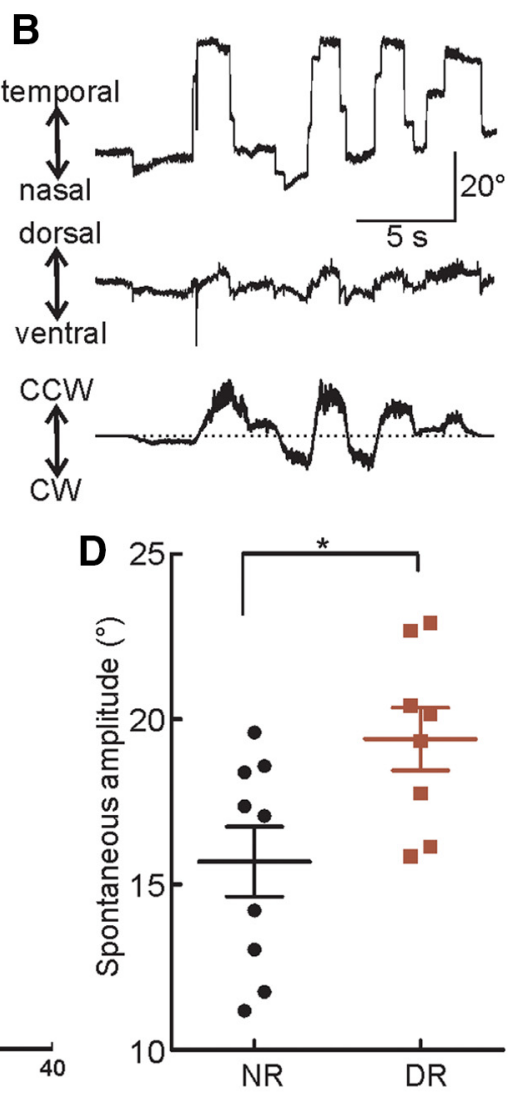

Figure 4. Larger self-generated saccades in DR mice. $\boldsymbol{A}$, A schematic diagram of the setup to study self-generated saccades in $(p<0.05$, $t$ test). Each point is the mean amplitude of spontaneous movements along the horizontal axis of one recording session (DR: $n=8$ from 2 mice; NR: $n=9$ from 3 mice). The population mean \pm SEM are also shown.

saccades evoked in DR mice were of larger amplitude and always temporally directed (contraversive) at all equivalent positions, including the anterior pole of the SC (Fig. 3A). No nasally directed ipsiversive saccades were evoked in the DR mice, even though the anterior SC represents nasal visual field in the superficial layers of both NR and DR mice (Fig. $3 A$ ). To further quantify this observation, for each animal we calculated the slope of the linear regression between the eye movement amplitudes and relative anterior-posterior separation of stimulation sites. Although the SC eye movement maps in the DR mice maintained normal polarity, they were "flatter" than the NR mice, i.e., having smaller slope $(28.0 \pm 2.7$ degrees $/ \mathrm{mm}$ in NR, $n=7 ; 12.8 \pm 2.1$ degrees/mm in DR, $n=7 ; p<0.001$; Fig. $3 B$ ).

The relatively larger amplitude of evoked saccades in DR mice suggests that the early development of SC eye movement map requires visual feedback to match motor output to overlying visual activity. To explore this further, we compared the amplitude of the self-generated spontaneous saccades in both NR and DR mice. Because we found that there were very few spontaneous eye movements under the body-restrained paradigm, we adopted a less restrained system (Dombeck et al., 2007) in which the headfixed mouse was able to run on a spherical treadmill (Fig. 4A). Under these more naturalistic conditions, the mice frequently made spontaneous eye movements while running, mostly along 
the nasotemporal axis, especially when they attempted to make turns (Fig. 4B). We calculated the mean amplitude of these saccades for each session of $\sim 2-10$ min on the apparatus. A session included 18-168 spontaneous saccades collected for both DR ( $n=2$ mice, eight sessions) and NR ( $n=3$ mice, nine sessions) groups. Just like the evoked saccades, the amplitude and peak velocity of the self-generated saccadic eye movements were correlated, demonstrating the typical main sequence (Fig. 4C). More importantly, the average eye movement amplitude was indeed larger in DR mice $(15.7 \pm 1.1$ degrees in NR, $n=9 ; 19.4 \pm 0.9$ degrees in DR, $n=8$; $p<0.05$; Fig. $4 D)$. This result is consistent with our observation that stimulation-evoked saccades in the DR mice are larger at equivalent points along the anterior-posterior axis of the SC (Fig. 3A). The differences between NR and DR groups suggest that visual experience during development is required to fine-tune the amplitude of saccades produced by a given location in the SC and to establish a correspondence between adjacent superficial and deep layer topographic representations.

\section{Discussion}

It had long been thought that rodents primarily use their heads rather than their eyes to orient toward salient stimuli (Schneider, 1969). However, with recent advances in high-speed video technology (Stahl, 2004) saccade-like eye movements have been documented in mice with amplitude and speed similar to that of other species (Sakatani and Isa, 2004, 2007). In this study we show that saccadic eye movements can be evoked by microstimulation in the SC, and the amplitudes of these eye movements depend on the stimulation locations. Our results suggest that eye movements in mice and their topographic representations may share similar circuit mechanisms with other well studied species. Given the abundance of genetic tools available in mice, our findings provide an important foundation for future studies using mice to investigate the organization and developmental of sensorimotor transformations in the SC.

In primates, the purpose of saccades is to target the fovea toward locations of interests in space for higher visual acuity. Although mice do not have a fovea, their RGCs are not uniformly distributed. A recent study showed that $\alpha$ RGCs display a nasotemporal gradient in density, size, and receptive fields, which would enhance sampling of the nasal visual fields (Bleckert et al., 2014). In addition, the part of the visual field adjacent to the vertical meridian is seen by both eyes $\left(\sim 60^{\circ}\right.$ visual space in mice), and binocular vision is known to increase visual acuity, contrast sensitivity, and depth perception (Campbell and Green, 1965; Parker, 2007). It is thus conceivable that mice make saccades to move their binocular visual field to achieve better sampling of certain regions of the visual space (Wallace et al., 2013).

We have found that there is clearly an eye movement map in the deep layers of the mouse SC with the same polarity as the retinotopic map in the superficial layers; however, it is difficult to quantify how precisely the two maps are aligned in mice because of the lack of fovea. It is known that the "magnification factor" of the retinotopic map in the superficial SC is $\sim 6-7$ degrees $/ 100$ $\mu \mathrm{m}$ (Cang et al., 2008). In comparison, we found that the difference of the saccade amplitudes was $\sim 4^{\circ}$ between two stimulation sites that were $100 \mu \mathrm{m}$ apart. This suggests that the retinotopic and eye movement maps may not be as precisely aligned in mice as in primates. It is likely that mice normally move both eyes and heads during natural orienting movements, just as primates do when they make larger gaze shifts (Gandhi and Katnani, 2011). A more natural setup, allowing both eye and head movements, will be needed in future studies to provide a more accurate quantification of the motor maps in the mouse SC.

In this paper, we have also initiated studies of factors influencing the development of SC eye movement maps. Our results of degraded motor maps in DR mice indicate that its development requires normal visual experience. In particular, the fact that DR mice exhibited larger saccades suggests that visual feedback is important for fine-tuning saccade precision and for aligning superficial and deep layer maps. Although the exact mechanisms remain unknown, this process likely involves a combination of refining connections between superficial and deep layers, as well as the calibration of the efficacy of deep layer output on brainstem oculomotor centers. Alternatively, it is conceivable that the visual map in the superficial SC is degraded by dark rearing, but this is unlikely as the visual map is established before vision onset and is experience independent (Cang and Feldheim, 2013). Furthermore, the evoked saccades were always contraversive in the DR mice, despite their normal ipsilateral representation, indicating a more likely misalignment between visual and motor maps. Finally, the degraded eye movement map in DR mice is reminiscent of findings of visuo-auditory map alignment in barn owls and ferrets. In those systems, the visual map in the SC or tectum is used as a topographic template to integrate visual spatial cues into the auditory space map, achieving visual and auditory map alignment in the presence of normal visual experience (King et al., 1996, 1998; Brainard and Knudsen, 1998). Similarly, neurons in the superficial and deeper SC are reciprocally connected (Isa and Hall, 2009), providing a potential neural substrate for visual input to instruct motor map development.

\section{References}

Bahill AT, Clark MR, Stark L (1975) The main sequence: a tool for studying human eye movements. Math Biosci 24:191-204. CrossRef

Bleckert A, Schwartz GW, Turner MH, Rieke F, Wong RO (2014) Visual space is represented by nonmatching topographies of distinct mouse retinal ganglion cell types. Curr Biol 24:310-315. CrossRef Medline

Brainard MS, Knudsen EI (1998) Sensitive periods for visual calibration of the auditory space map in the barn owl optic tectum. J Neurosci 18:39293942. Medline

Campbell FW, Green DG (1965) Monocular versus binocular visual acuity. Nature 208:191-192. CrossRef Medline

Cang J, Feldheim DA (2013) Developmental mechanisms of topographic map formation and alignment. Annu Rev Neurosci 36:51-77. CrossRef Medline

Cang J, Wang L, Stryker MP, Feldheim DA (2008) Roles of ephrin-as and structured activity in the development of functional maps in the superior colliculus. J Neurosci 28:11015-11023. CrossRef Medline

Dhande OS, Estevez ME, Quattrochi LE, El-Danaf RN, Nguyen PL, Berson DM, Huberman AD (2013) Genetic dissection of retinal inputs to brainstem nuclei controlling image stabilization. J Neurosci 33:17797-17813. CrossRef Medline

Dias EC, Segraves MA (1999) Muscimol-induced inactivation of monkey frontal eye field: effects on visually and memory-guided saccades. J Neurophysiol 81:2191-2214. Medline

Dombeck DA, Khabbaz AN, Collman F, Adelman TL, Tank DW (2007) Imaging large-scale neural activity with cellular resolution in awake, mobile mice. Neuron 56:43-57. CrossRef Medline

Dräger UC, Hubel DH (1976) Topography of visual and somatosensory projections to mouse superior colliculus. J Neurophysiol 39:91-101. Medline

du Lac S, Knudsen EI (1991) Early visual deprivation results in a degraded motor map in the optic tectum of barn owls. Proc Natl Acad Sci U S A 88:3426-3430. CrossRef Medline

Gandhi NJ, Katnani HA (2011) Motor functions of the superior colliculus. Annu Rev Neurosci 34:205-231. CrossRef Medline

Huberman AD, Niell CM (2011) What can mice tell us about how vision works? Trends Neurosci 34:464-473. CrossRef Medline 
Isa T, Hall WC (2009) Exploring the superior colliculus in vitro. J Neurophysiol 102:2581-2593. CrossRef Medline

King AJ, Schnupp JW, Carlile S, Smith AL, Thompson ID (1996) The development of topographically-aligned maps of visual and auditory space in the superior colliculus. Prog Brain Res 112:335-350. CrossRef Medline

King AJ, Schnupp JW, Thompson ID (1998) Signals from the superficial layers of the superior colliculus enable the development of the auditory space map in the deeper layers. J Neurosci 18:9394-9408. Medline

Parker AJ (2007) Binocular depth perception and the cerebral cortex. Nat Rev Neurosci 8:379-391. CrossRef Medline

Sakatani T, Isa T (2004) PC-based high-speed video-oculography for measuring rapid eye movements in mice. Neurosci Res 49:123-131. CrossRef Medline

Sakatani T, Isa T (2007) Quantitative analysis of spontaneous saccade-like rapid eye movements in C57BL/6 mice. Neurosci Res 58:324-331. CrossRef Medline

Schiller PH, Stryker M (1972) Single-unit recording and stimulation in superior colliculus of the alert rhesus monkey. J Neurophysiol 35:915-924. Medline
Schneider GE (1969) Two visual systems. Science 163:895-902. CrossRef Medline Stahl JS (2004) Using eye movements to assess brain function in mice. Vision Res 44:3401-3410. CrossRef Medline

Stein BE (1984) Development of the superior colliculus. Annu Rev Neurosci 7:95-125. CrossRef Medline

Wallace DJ, Greenberg DS, Sawinski J, Rulla S, Notaro G, Kerr JN (2013) Rats maintain an overhead binocular field at the expense of constant fusion. Nature 498:65-69. CrossRef Medline

Wang L, Sarnaik R, Rangarajan K, Liu X, Cang J (2010) Visual receptive field properties of neurons in the superficial superior colliculus of the mouse. J Neurosci 30:16573-16584. CrossRef Medline

Yonehara K, Ishikane H, Sakuta H, Shintani T, Nakamura-Yonehara K, Kamiji NL, Usui S, Noda M (2009) Identification of retinal ganglion cells and their projections involved in central transmission of information about upward and downward image motion. PLoS One 4:e4320. CrossRef Medline 